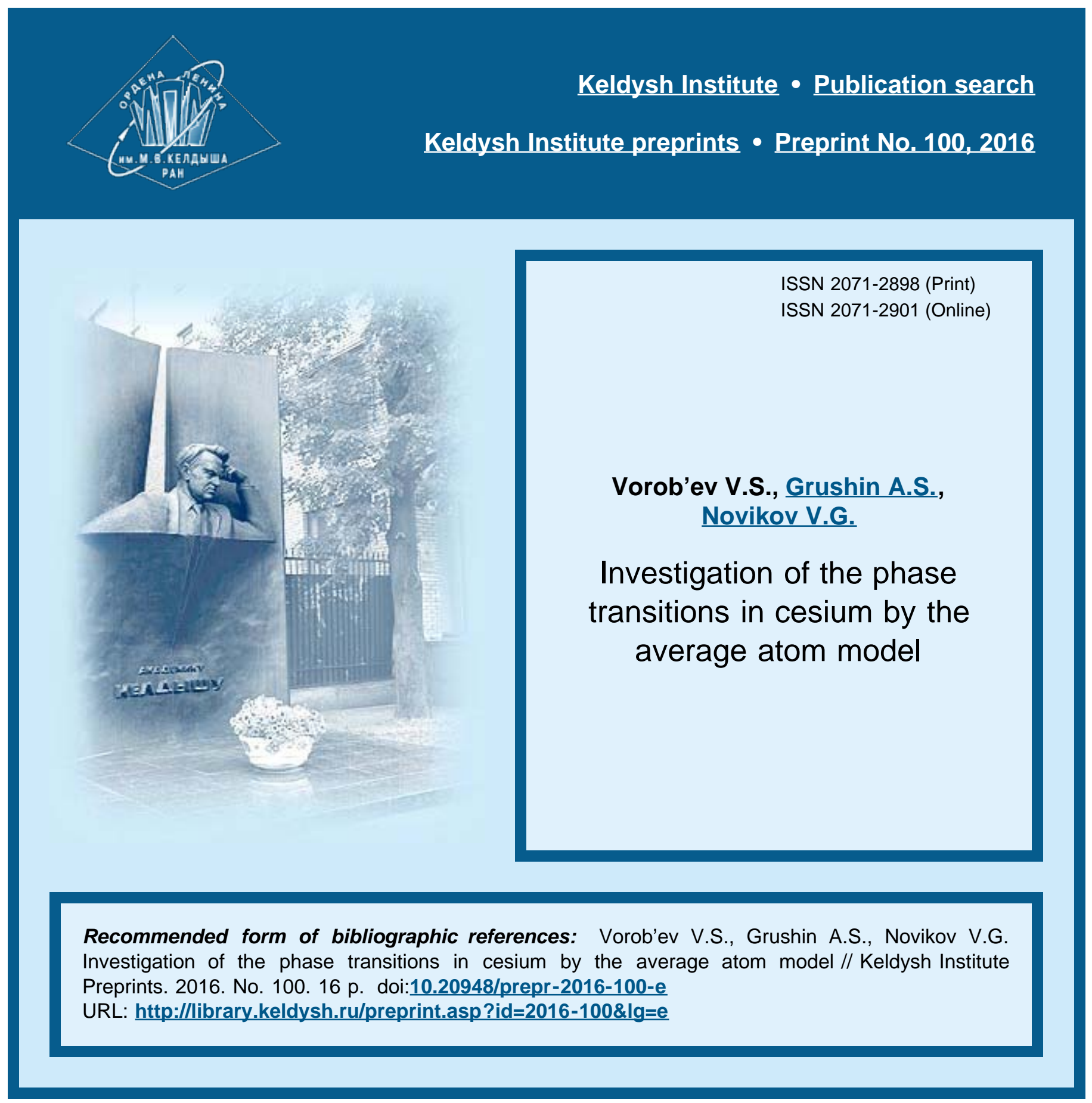




\author{
Ор д е н а Л е н и н а \\ ИНСТИТУТ ПРИКЛАДНОЙ МАТЕМАТИКИ \\ имени М.В. Келдыша \\ Российской академии наук
}

V.S. Vorob'ev, A.S. Grushin, V.G. Novikov

\title{
Investigation of the phase transitions in cesium by the average atom model
}


Воробъёв В.С., Грушин А.С., Новиков В.Г.

Исследование фазовых переходов в цезии с помощью модели среднего атома

В данной работе, используя модель среднего атома (квазизонная модель вещества), основанную на модели Хартри-Фока-Слэтера, показано, что кривая холодного сжатия цезия имеет два локальных минимума в давлении - первый минимум находится при относительно малых плотностях $0.3 \Gamma / \mathrm{cm}^{3}$, и второй

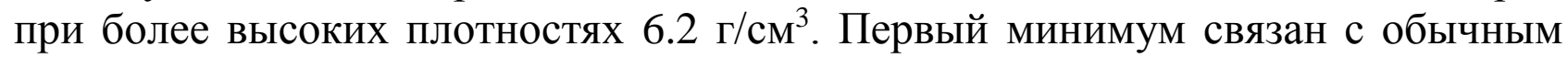
фазовым переходом жидкость-пар в области критической точки, параметры которого хорошо изучены, в том числе - экспериментально. Второй минимум давления, обусловленный обменом валентными электронами между $\mathrm{s}$ и d подуровнями, приводит ко второму, плазменному, фазовому переходу с

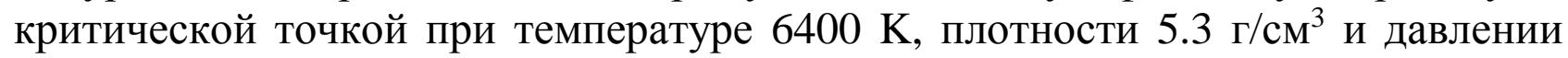
порядка 61000 атм. При этом, второй фазовый переход, наблюдаемый в вырожденной неидеальной плазме, сопровождается резким изменением среднего заряда со значения 1.5 при плотности 3 г/см ${ }^{3}$ до значения 3.5 при

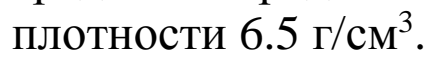

Ключевые слова: уравнение состояния, фазовый переход, физика плазмы, модель среднего атома, цезий, неидеальная плазма

Работа выполнена при поддержке гранта РНФ № 14-11-00699. Расчёты проводились на СЭВМ К-100 (ИПМ им. М.В. Келдыша РАН).

Vladimir Sergeevich Vorob'ev, Alexander Sergeevich Grushin, Vladimir Grigor'evich Novikov

\section{Investigation of the phase transitions in cesium by the average atom model}

Using the average atom method (quasizone model) we show that the cesium cold curve has two minimums - the first one is at relatively small densities $0.3 \mathrm{~g} / \mathrm{cm}^{3}$ and the second one is at higher densities $6.2 \mathrm{~g} / \mathrm{cm}^{3}$. The first one leads to the usual critical point characterizing the liquid-vapor phase transition, which is confirmed by experimental data. The reason for the second minimum is connected with s-d transition of valence electron. This leads to a second phase transition with the critical point at temperature $6400 \mathrm{~K}$, density $5.3 \mathrm{~g} / \mathrm{cm}^{3}$ and pressure approximately 61000 atm. This phase transition takes place in the cesium plasma state. In this degenerate nonideal plasma with mean ion charge 1.5 and density $3 \mathrm{~g} / \mathrm{cm}^{3}$ jumps in a highly ionized state with mean charge of 3.5 and density $6.5 \mathrm{~g} / \mathrm{cm}^{3}$.

Key words: equation of state, phase transition, physics of plasma, average atom model, cesium, nonideal plasma

This work was supported by the Russian Science Foundation through Grant No. 14-11-00699. Calculations have been performed at K-100 supercomputer (KIAM RAS). 


\section{Introduction}

Properties of matter at ultra-high pressures have always been a significant interest in a number of areas of physics and related sciences - astrophysics, geophysics, and some applied sciences [1]. In recent years, these studies have purchased especially large interest. This is due to the development of new experimental methods [2-6], using powerful current generators and lasers with ultrashort pulse duration. With their help it is possible to create record high pressures, which could not be obtained by conventional explosive installations or easy gas guns. The densities achieved in these experiments, several times higher than the density under normal conditions, leading to substantial changes in the nuclear, chemical and electronic structure of the materials. This may also cause previously unknown phase transformations. For describing of the physical processes and phenomena in these conditions, it is necessary to have mathematical models of the state of matter. Particularly, the chemical [4-6] and quantum-statistical models [7-9] are used.

In the first case, a dense matter is treated as an equilibrium mixture of molecules, atoms, molecular and atomic ions, and electrons. The equilibrium composition of the mixture follows from the minimum of free energy. However, consistently take into account the deformation of the electron shells of the particles, the ejection of discrete levels in the continuous spectrum, caused by high pressure, formation of new phase transitions in the framework of chemical models is difficult. Here are preferred quantum-statistical cell models with boundary conditions, borrowed from the theory of solids. One such model is the average atom (AA) model [7-10]. This is a spherical cell model, combined with a self-consistent approximation of the density functional and Bloch boundary conditions. Its peculiarity lies in the fact that, along with bound electrons localized within the atomic cell (Wigner-Seitz), and the states of the continuous spectrum, which are applicable to semi-classical description, there are more electrons in the allowed energy bands (quasi-zones). A detailed account of these states is necessary for the correct description of pressure ionization - the destruction of the outer electron shells and squeezing the discrete levels in the continuum due to the interaction of atoms in condensed matter. Therefore the average atom (AA) model is sometimes called the quasizone model (QZM).

In this paper, the average atom model is used to calculate the thermodynamic functions of cesium both at relatively low and high pressure. The choice of this element is not accidental. This is one of the few metals for which the parameters of the liquid-vapor phase transition were measured $[11 ; 12]$. In addition, a number of studies $[13 ; 14]$ have suggested that an increase in temperature and pressure leads to one more phase transition. To verify this prediction by quantum-statistical calculation of the thermodynamic functions of the cesium is one of the goals of this work.

First, a brief description of the main provisions of the average atom model (a more detailed description can be found in [8]) is given. Then we present the results of calculations. They confirmed the presence of first order phase transition at densities 
lower than $1 \mathrm{~g} / \mathrm{cm}^{3}$. This phase transition on the parameters corresponds to the usual liquid-gas transition, measured in references $[11 ; 12]$. This was confirmed by the use of AA model for describing phase transitions in real substance. At higher temperatures and densities was found the second phase transition, in which the cesium atom is significantly ionized. Its cause is s-d transition of valence electron. This fact as noted earlier led to the anomalies in the cesium melting curve [15-18]. The values of the characteristic parameters of degeneration and interaction are given along the lines of phase equilibrium. The peculiarities of the band structure of the levels of the cesium atom are discussed, leading to the appearance of a minimum on the cold-pressure curve and, consequently, the appearance of the plasma phase transition.

\section{The fundamentals of the quasizone model}

In the quasizone model one assumes that each atomic nucleus with charge $Z$ is placed at the center of the Wigner-Seitz sphere defined by the matter density $\rho$ and an atomic weight of the substance $A$. All $Z$ electrons of the atom are also in the cell, making it electrically neutral. We assume in the quasizone model the most general boundary conditions for the wave function - the conditions resulting from translational symmetry, i.e. Bloch-type conditions. At the boundary of the spherical cell $r=r_{0}$ for the wave function $\Psi_{\gamma}(\vec{r})$ we have $\left|\Psi_{\gamma}(\vec{r})\right|^{2}=\left|\Psi_{\gamma}(-\vec{r})\right|^{2}$ or $\Psi_{\gamma}(\vec{r})=e^{i \vec{k} \vec{r}} \Phi_{\gamma \vec{k}}(\vec{r})$, where $\gamma$ is the set of quantum numbers for one-electron state, $\vec{k}$ is an arbitrary real constant vector (quasi-momentum) and $\Phi_{\gamma \vec{k}}(\vec{r})$ is a periodic function.

For the function $\Psi_{\gamma}(\vec{r})$ using continuity of its derivative at $|\vec{r}|=r_{0}$ we obtain

$$
\left\{\begin{array}{c}
e^{-i \vec{k} \vec{r}} \Psi_{\gamma}(\vec{r})=e^{i \vec{k} \vec{r}} \Psi_{\gamma}(-\vec{r}) \\
e^{-i \vec{k} \vec{r}} \frac{\partial}{\partial r} \Psi_{\gamma}(\vec{r})=-e^{i \vec{k} r} \frac{\partial}{\partial r} \Psi_{\gamma}(-\vec{r})
\end{array}\right.
$$

Here the effective reciprocal cell in the $\vec{k}$-space is also spherical in this approximation, and its volume is equal to $4(2 \pi)^{3} / 3 \pi r_{0}^{3}$. It follows that the quantity $|\vec{k}| r_{0}$, henceforth denoted by $k$, can take values in the interval $0 \leq k \leq k_{0}=\left(\frac{9 \pi}{2}\right)^{1 / 3} \approx 2.418$.

Assuming the self-consistent potential spherically symmetric, we get in nonrelativistic approximation the electron wave function as a superposition of partial solutions of Schrödinger equation 


$$
\Psi_{\gamma}(\vec{r})=\sum_{\ell^{\prime} \geq m} i^{\ell^{\prime}} A_{n \ell m, \ell^{\prime}}(k) \frac{1}{r} R_{\varepsilon \ell^{\prime}}(r) Y_{\ell^{\prime} m}(\vartheta, \varphi)
$$

with $\gamma=n \ell m$, radial wave functions $R_{\varepsilon \ell}(r)$, spherical harmonics $Y_{\ell^{\prime} m}(\vartheta, \varphi)$, energy $\varepsilon=\varepsilon_{n \ell m}(k)$ and the projection $m$ of the orbital momentum $\ell^{\prime}$ on the $z$-axis, which is directed along the quasi-momentum vector $\vec{k}$. Here the numbers $n$ and $\ell$ determine the energy level to which the band shrinks when the density of matter is decreased; the factor $i^{\ell^{\prime}}$ is introduced to make the expansion coefficients $A_{n \ell m, \ell^{\prime}}(k)$ real. The $A_{n \ell m, \ell^{\prime}}(k)$ are calculated from the boundary conditions.

In quasizone model when increasing the density of matter instead of bound levels we get the energy bands. As a result one obtains the smooth dependence of thermodynamic and optical properties over temperature and density. The correct description of the spectrum structure allows one to obtain reasonable thermodynamic characteristics of dense plasma in a wide range of temperature and density.

\section{Results of calculations and discussion}

The cold curve and two pressure isotherms $\mathrm{T}=1938$ and $\mathrm{T}=5620 \mathrm{~K}$, calculated by using QZM approach, are presented in Fig. 1. First of all, note that the cold curve has two minimums - the first one is at relatively small densities $\rho \sim 0.3 \mathrm{~g} / \mathrm{cm}^{3}$ and second is at higher densities $\rho \sim 6.2 \mathrm{~g} / \mathrm{cm}^{3}$. Their causes will be discussed below. Accordingly, at temperatures of $\mathrm{T}=1938$ and $6460 \mathrm{~K}$, there are two critical isotherms. Let us consider each of these isotherms in more details.

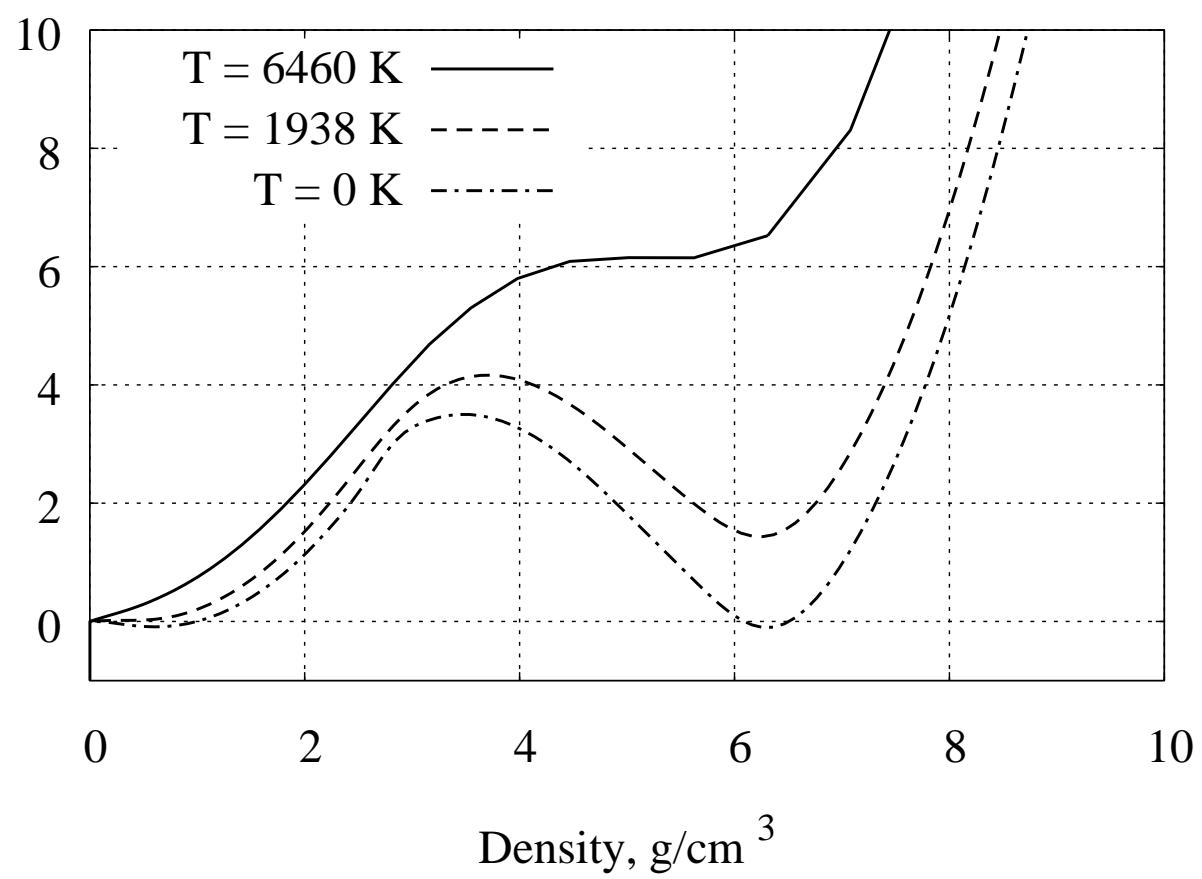

Fig. 1. Pressure isotherms for cesium at $\mathrm{T}=0$ (cold), $1938 \mathrm{~K}$ and $6460 \mathrm{~K}$. 
The temperature $\mathrm{T}=1938 \mathrm{~K}$ is not chosen casually. It corresponds to the usual measurements $[11 ; 12]$ of the critical temperature of the liquid-vapor in cesium. This critical transition region is represented on a larger scale in Fig. 2. The values of the critical density and pressure along this isotherm are equal to $0.35 \mathrm{~g} / \mathrm{cm}^{3}$ and $180 \mathrm{~atm}$ correspondingly. This density is close to experimental one $\left(0.39 \mathrm{~g} / \mathrm{cm}^{3}[12]\right)$. The pressure is higher than the experimental value (94 atm [12]). It should be noted that the accuracy of AA model decreases with decreasing density. In particular, the loss of accuracy in the calculation of the critical pressure due to the fact that the critical pressure is the small difference between two large terms - thermal and potential pressure contributions. Despite some uncertainty in the determination of the critical pressure, the first phase transition parameters calculated by AA model corresponds to the conventional transition liquid - vapor, which is confirmed by experimental data $[11 ; 12]$. This suggests the possibility of using this method for determining the parameters of phase transitions even in the border area of its application. As a rule, with the increase of density the precision of the AA model grows and so does the reliability of its predictions.

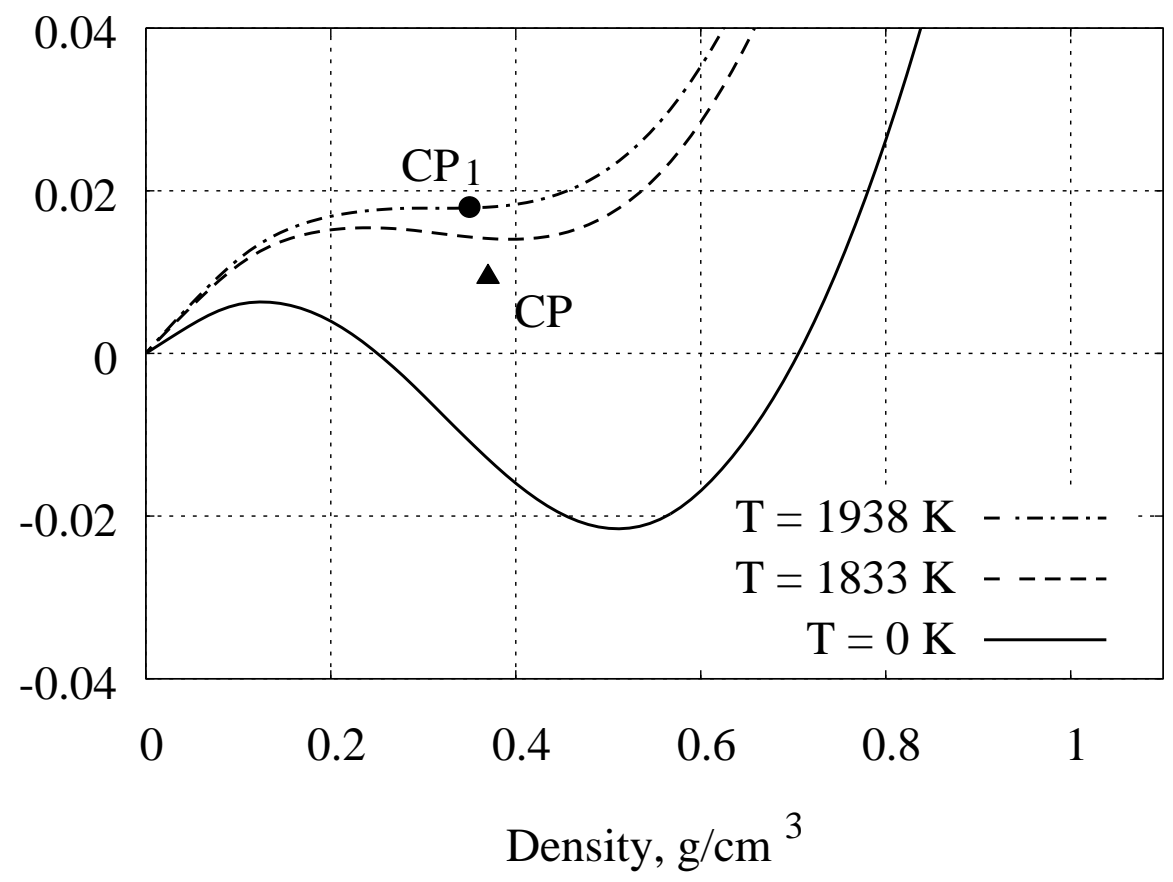

Fig. 2. The region of the liquid-gas critical point at the density-pressure plane.

$\mathrm{CP}$ and $\mathrm{CP}_{1}$ are the experimental [14] and calculated in this work values of the liquid-gas critical point.

Second critical transition region is represented on a larger scale in Fig. 3. 


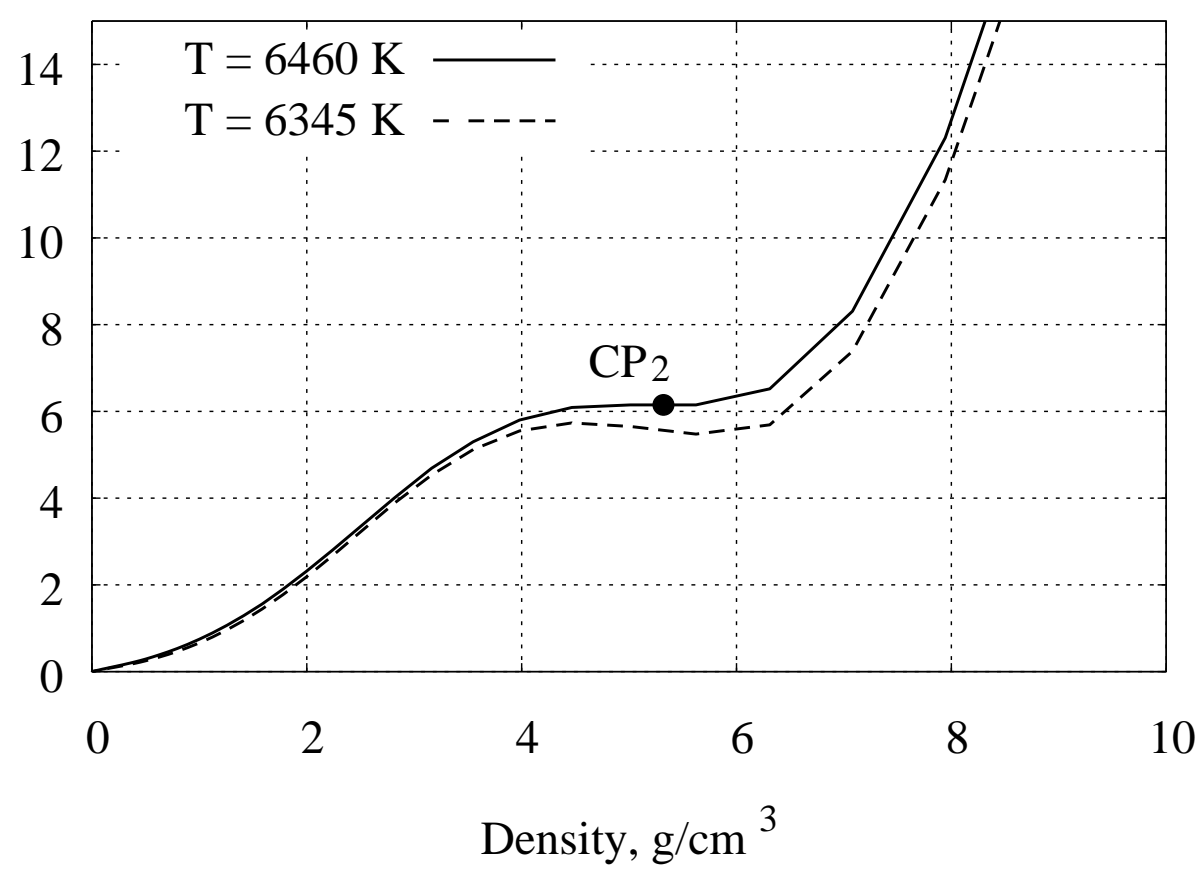

Fig. 3. The plasma critical and near critical isotherms at the density-pressure plane.

$\mathrm{CP}_{2}$ is the critical point at high temperature and high pressure.

The critical point parameters are $\mathrm{T}_{\mathrm{c}}=6460 \mathrm{~K}, \rho_{\mathrm{c}}=5.32 \mathrm{~g} / \mathrm{cm}^{3}$ and $\mathrm{P}_{\mathrm{c}}=6.15 \mathrm{GPa}$. In the presence of the set of isotherms with a characteristic van der Waals loop, we can construct curves of phase equilibrium using, for example, Maxwell equal area rule or other standard methods. Such curves are presented in Figs. 4, 5. The cesium phase diagram at the temperature-density plane is shown in Fig. 4.

Cesium phase diagram at low densities and temperatures has been investigated in a number of works [15-22]. Fig. 4 shows the experimental values of the liquid-gas binodal [12] and the melting line [18] only. The calculated values of the binodal are very close to the experimental ones, and they are not shown in this figure. The inscription "new phase" on the figure correspond to a new binodal, which is realized at higher densities and higher temperatures and has the critical parameters $\rho_{\mathrm{c}}=5.32$ $\mathrm{g} / \mathrm{cm}^{3}$ and $\mathrm{T}_{\mathrm{c}}=6460 \mathrm{~K}$. The pressure-temperature phase diagram is shown in Fig. 5. 


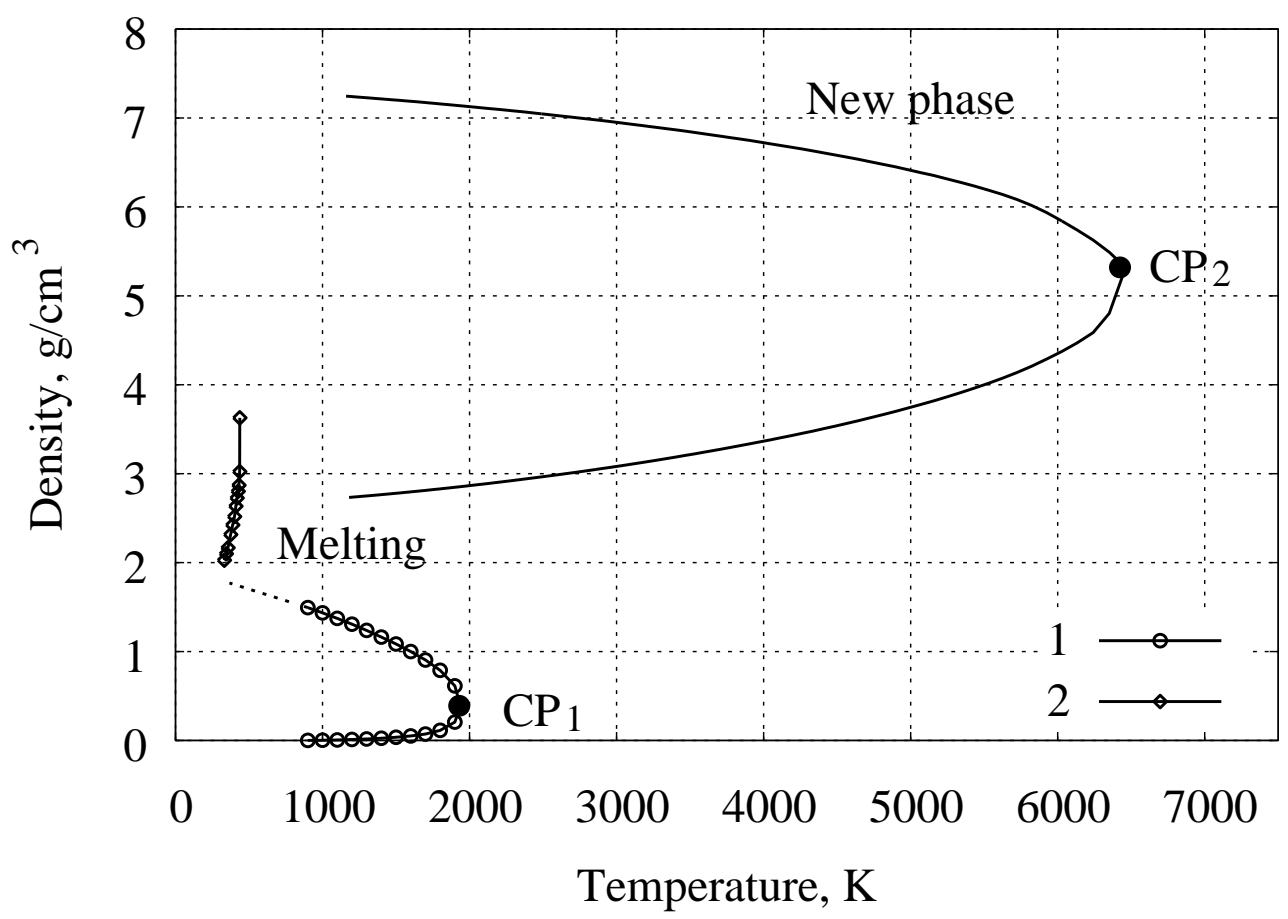

Fig. 4. Density-temperature cesium phase diagram. Experimental data: 1 - [14], 2 - [18].

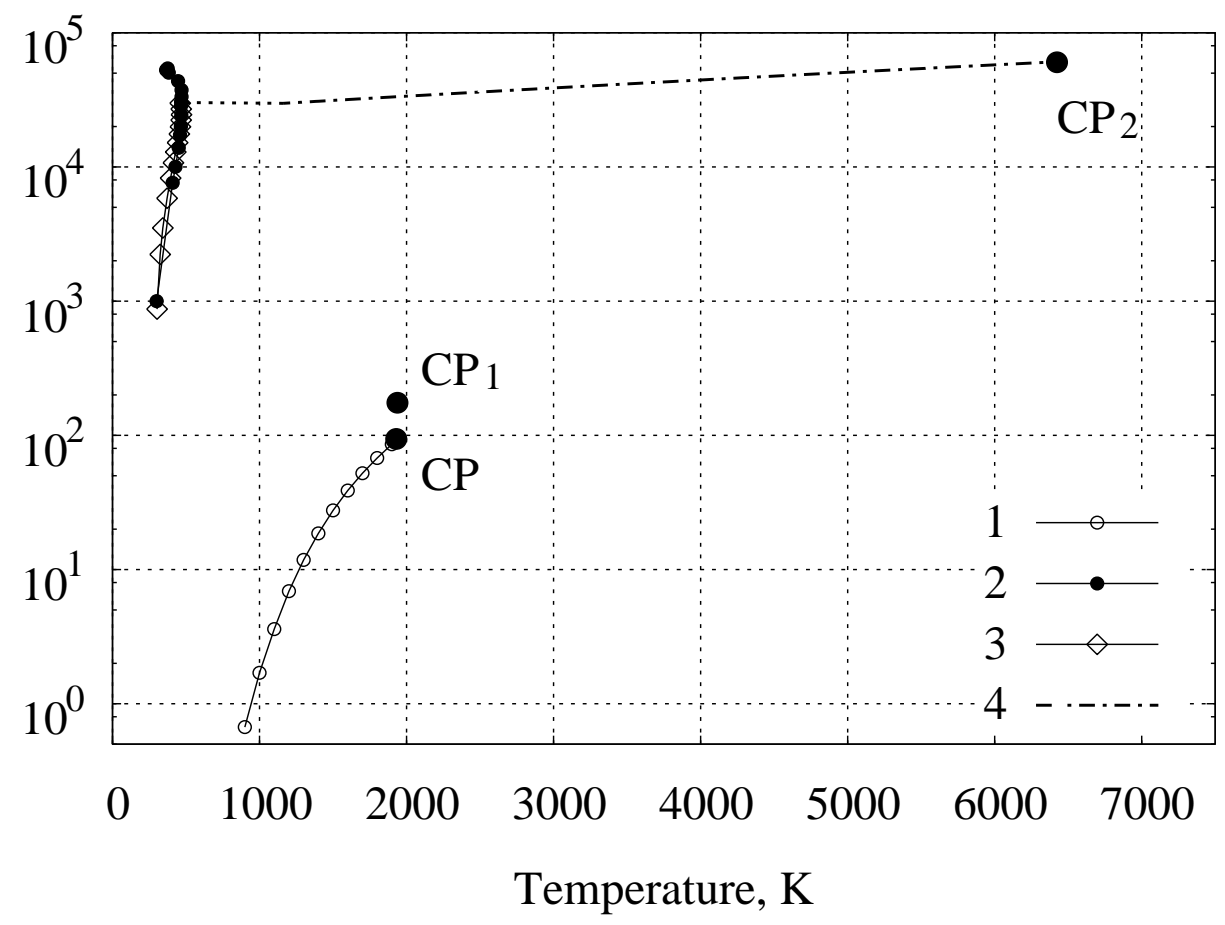

Fig. 5. Pressure-temperature cesium phase diagram. Experimental data: $1-$ is the liquid-gas phase curve [14], $2-[19]$ and $3-[18]$ is the melting curve. 4 is the calculated in this work phase diagram.

The remaining notation is the same as in Figs. $1-4$. 
We emphasize particularly the cesium melting curve marked in the works [16; 18]. In cesium when pressure becomes more than 40000 atm the normal melting turns into abnormal. According to [18] it is associated with the transition of the valence electron from $\mathrm{s}$ into $\mathrm{d}$-state. There is reason to believe that similar transition also occurs in liquid cesium. Below we shall discuss this in more details. Importantly, the pressure of the new phase (line 4) with decreasing temperature tends to the area in which the normal melting becomes abnormal.

In order to understand what a new phase is, let us consider in more detail its properties. The ionization composition and average ion charge along the phase lines are presented in figures 6 and 7. These figures show that cesium atoms are significantly ionized on both branches of phase equilibrium line. The average ion charge is more than 1.5 and less than 3.5 both on the lower and top branches of the phase curve. Ion density is less than 2.4 times the electron density at the critical temperature. With decreasing temperature, this difference increases. In fact, we have plasma of ions with charge $\mathrm{Z}$ and electrons, in which the phase transition becomes from a state with a smaller ion charge in a state with greater ion charge. The parameters of this plasma are such that he electron gas is strongly degenerate at such conditions. The degenerate parameter is $\eta=N_{e}^{1 / 3} \Lambda_{e}$, where $\Lambda_{e}=h / \sqrt{2 \pi m T}$ is the electron thermal wave length. The values of this parameter along the phase line are shown in Fig. 8 and they are everywhere larger than unity.

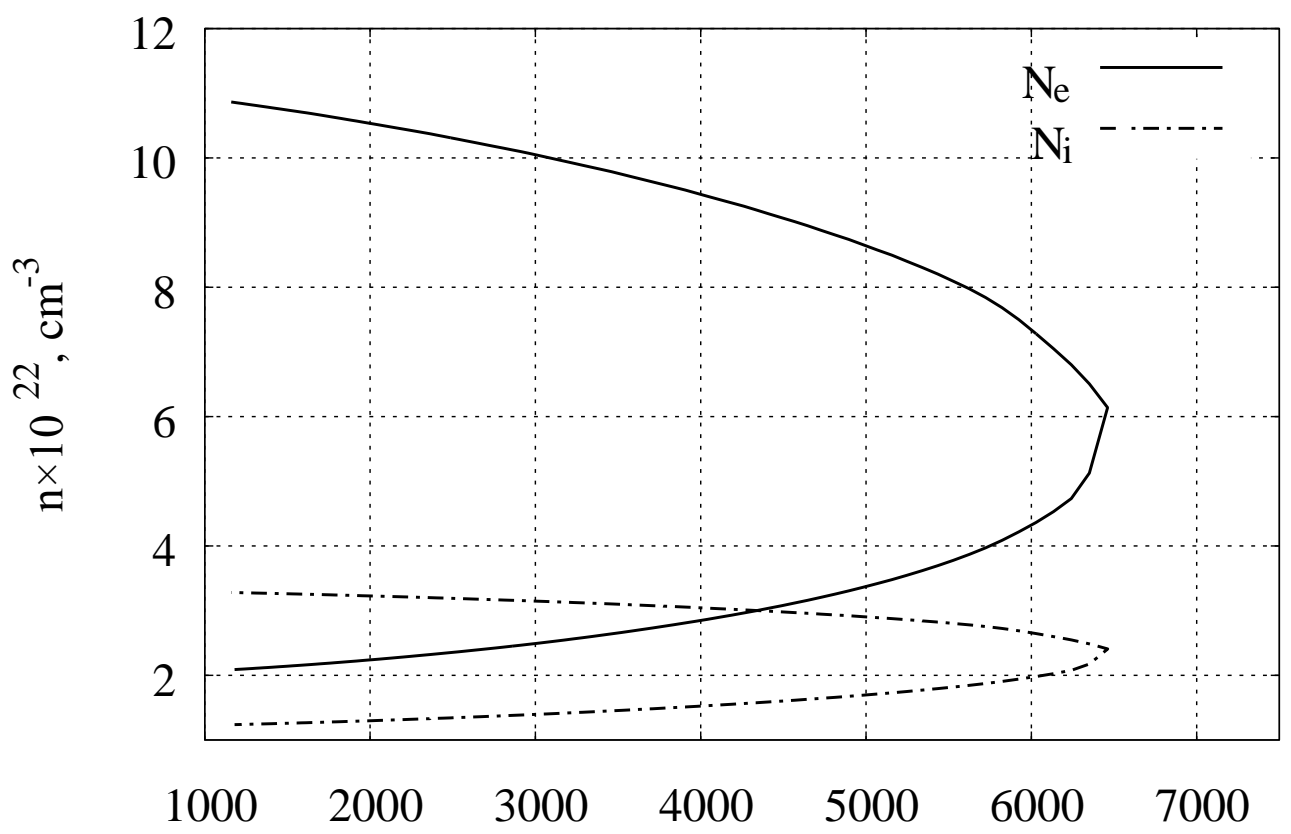

Temperature, $\mathrm{K}$

Fig. 6. Dependencies of electron and ion densities along the phase equilibrium line.

$\mathrm{N}_{\mathrm{e}}, \mathrm{N}_{\mathrm{i}}$ are the densities of electrons and ions respectively. 


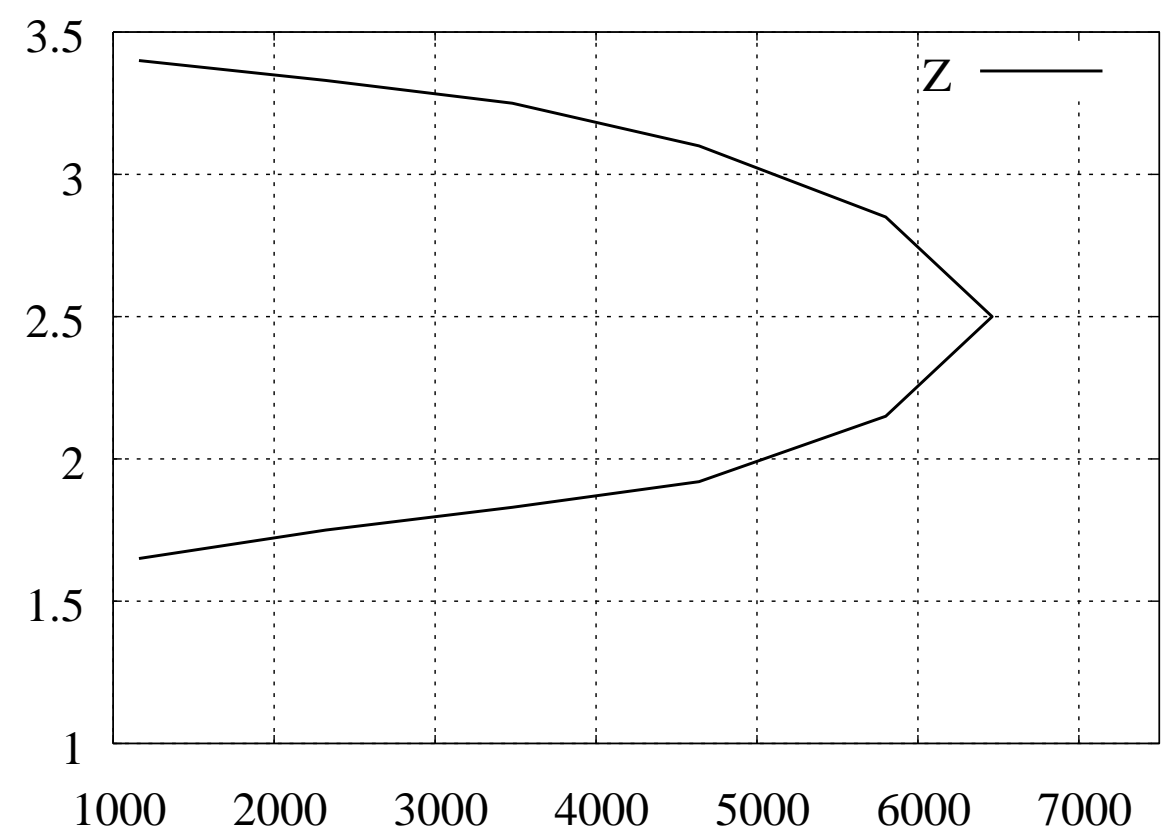

Temperature, $\mathrm{K}$

Fig. 7. Mean ion charge along the phase line.

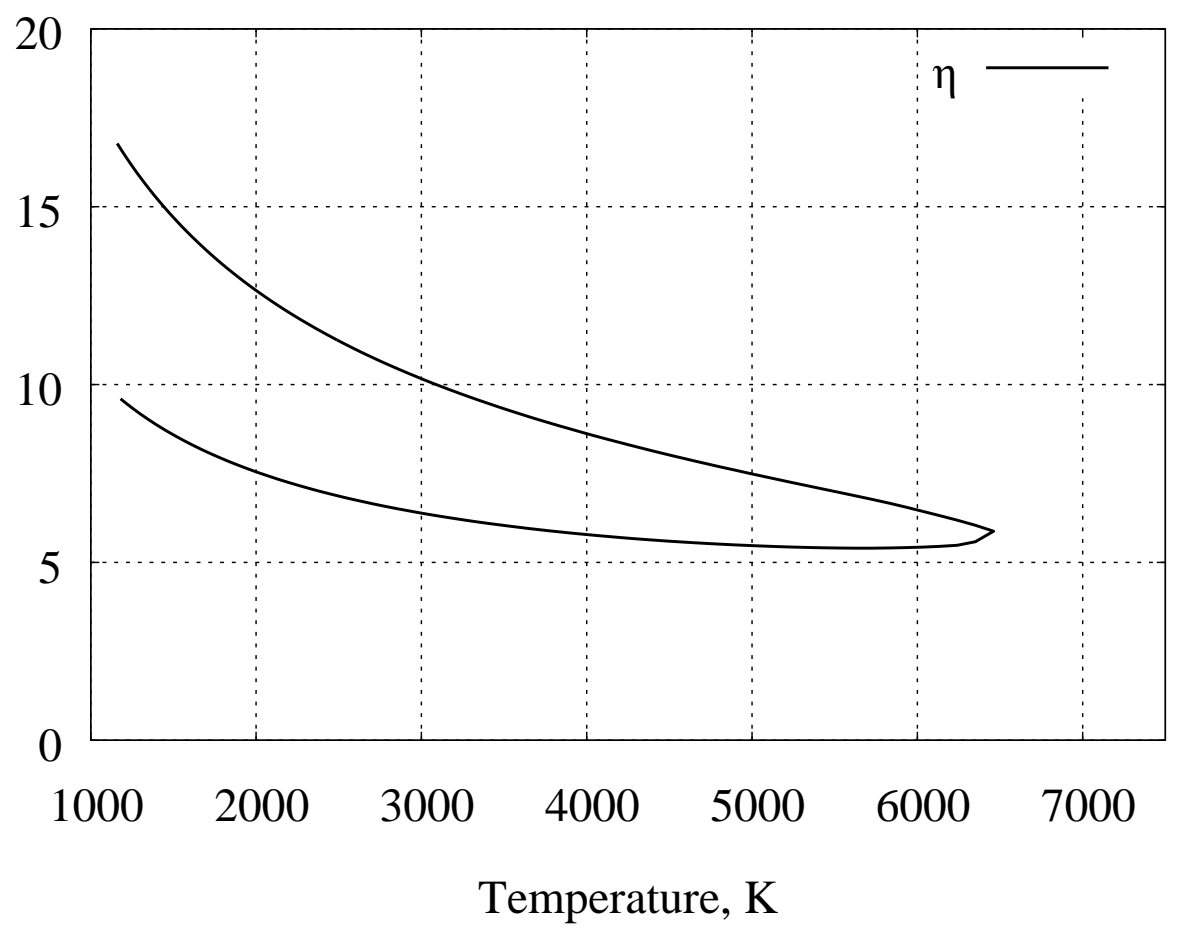

Fig. 8. The values of the electron degenerate parameter $\eta$ along the phase line.

It is of interest to find out what is non-ideality of the considered plasma state. The system under consideration is characterized by three parameters of interaction: electron-electron, electron-ion and ion-ion 


$$
\gamma_{e e}=\left(3 \pi^{2} N_{e}\right)^{2 / 3} \hbar^{2} / 2 m T \quad ; \quad \gamma_{e Z}=Z e^{2} N_{i}^{1 / 3} / T \quad ; \quad \gamma_{Z Z}=Z^{2} e^{2} N_{i}^{1 / 3} / T
$$

The corresponding values of the interaction parameters along the phase line are shown in Fig. 9.

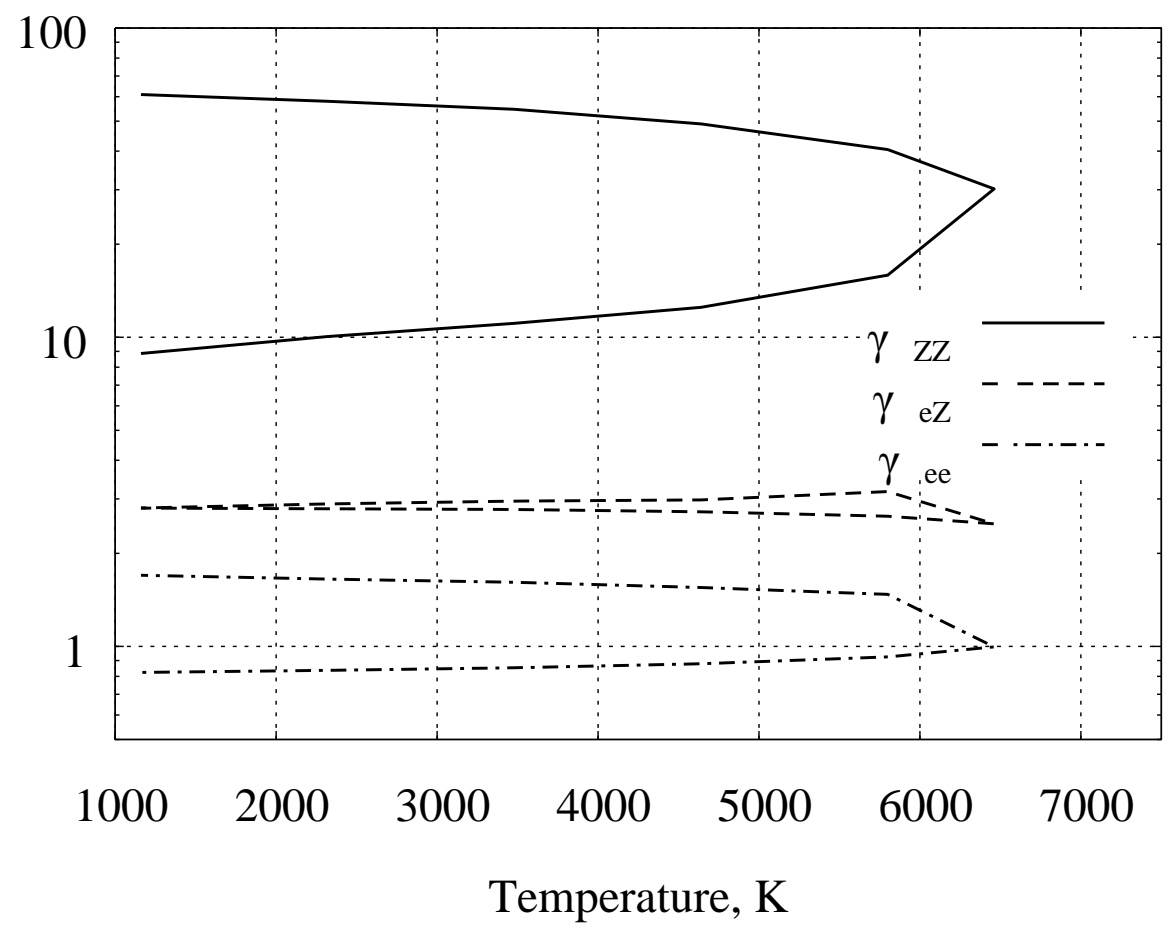

Fig. 9. The values of the interaction parameters along the phase line.

According to this picture, the interaction of electrons characterized by the parameter $\gamma_{e e} \sim 1$. The interaction of the electron-ion is stronger and appropriate parameter is $\gamma_{e Z} \sim 3$. The strongest is the interaction between the ions as $\gamma_{Z Z}>10$.

So in the domain of the second phase transition we have strongly interacted plasma with degenerate electrons.

An important feature is the cohesive energy of the atoms. It is defined as $U_{c o h}=U(0, \rho)-U_{0}$. Here, from the total energy of the cell at zero temperature and fixed density is subtracted corresponding cell energy at zero temperature and density. The value of $U_{0}$ is total ionization energy of cesium atom, i.e. the energy required to remove all electrons from a neutral atom. The cesium cohesive energy has been calculated by different methods in a number of works [20-22]. 


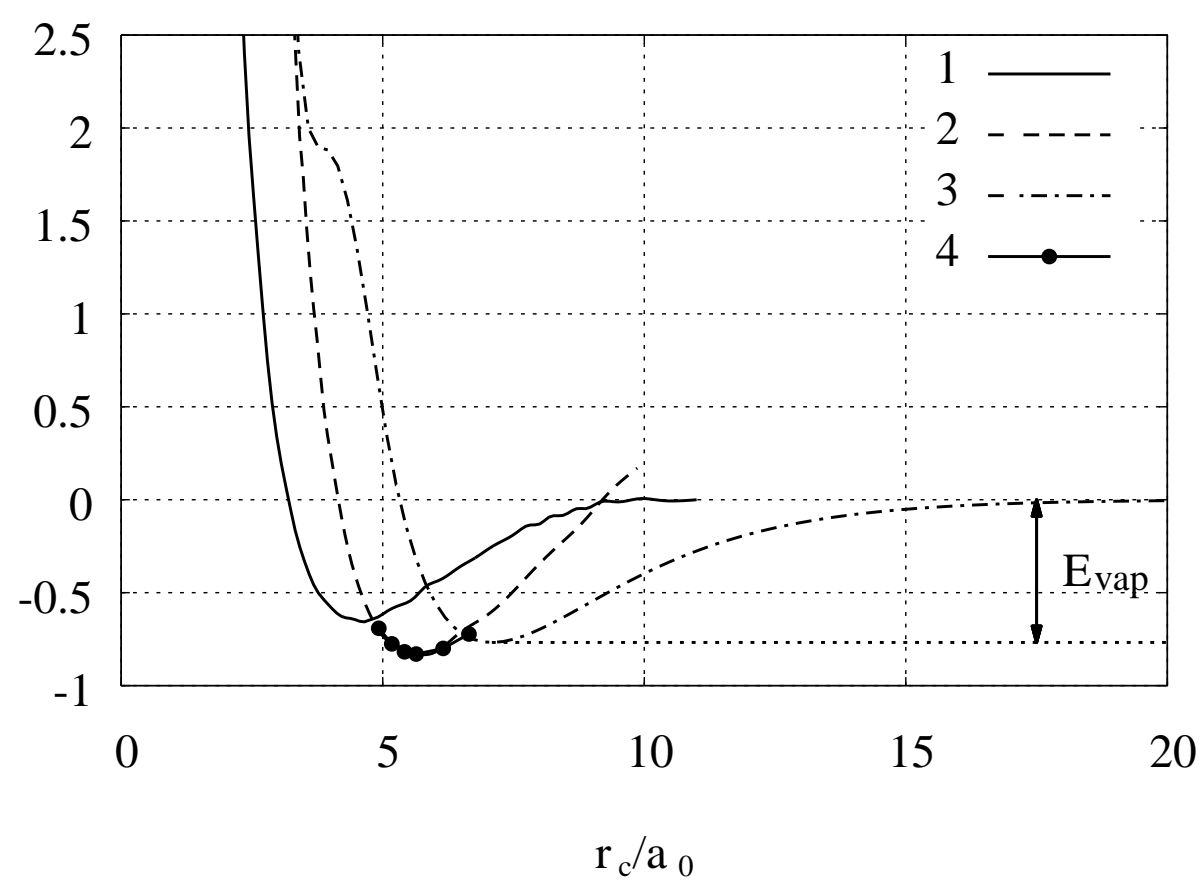

Fig. 10. Cohesive energy of cesium depends on the cell radius (atomic units).

Calculations: 1 - [21], 2 - [20], 3 - this work, 4 - [19].

The value $\mathrm{E}_{\mathrm{vap}}$ is the heat of vaporization.

As it can be seen from Fig. 10, all the curves have a minimum depth close to the heat of evaporation. However, the position of this minimum depends on the nature of the repulsion at short distances. In Ref. [22] to account for the interaction of the valence electron with the ionic core used Kratzer potential, providing the softest repulsion. In Ref. [21] the repulsion ensured the Madelung energy of point ions and the exchange energy of a homogeneous electron gas $E_{e x c}=-0.458 / r_{s}$. In this paper, the concept of point ion is not used, which effectively led to a stronger repulsion. In contrast to [20-22] on the repulsive branch of our energy curve is visible feature with the inflection point, the presence of which ensures the coexistence of a second minimum for cold-pressure curve (see Fig. 1). The band structure of levels depending on the density at zero temperature is shown in Fig. 11.

In order to determine the cause of the second minimum in the cold curve and thus the appearance of the second critical point, consider the band structure of most of the low-lying cesium atom levels. According to our model, atomic levels are turned into bands with increase of the density. 


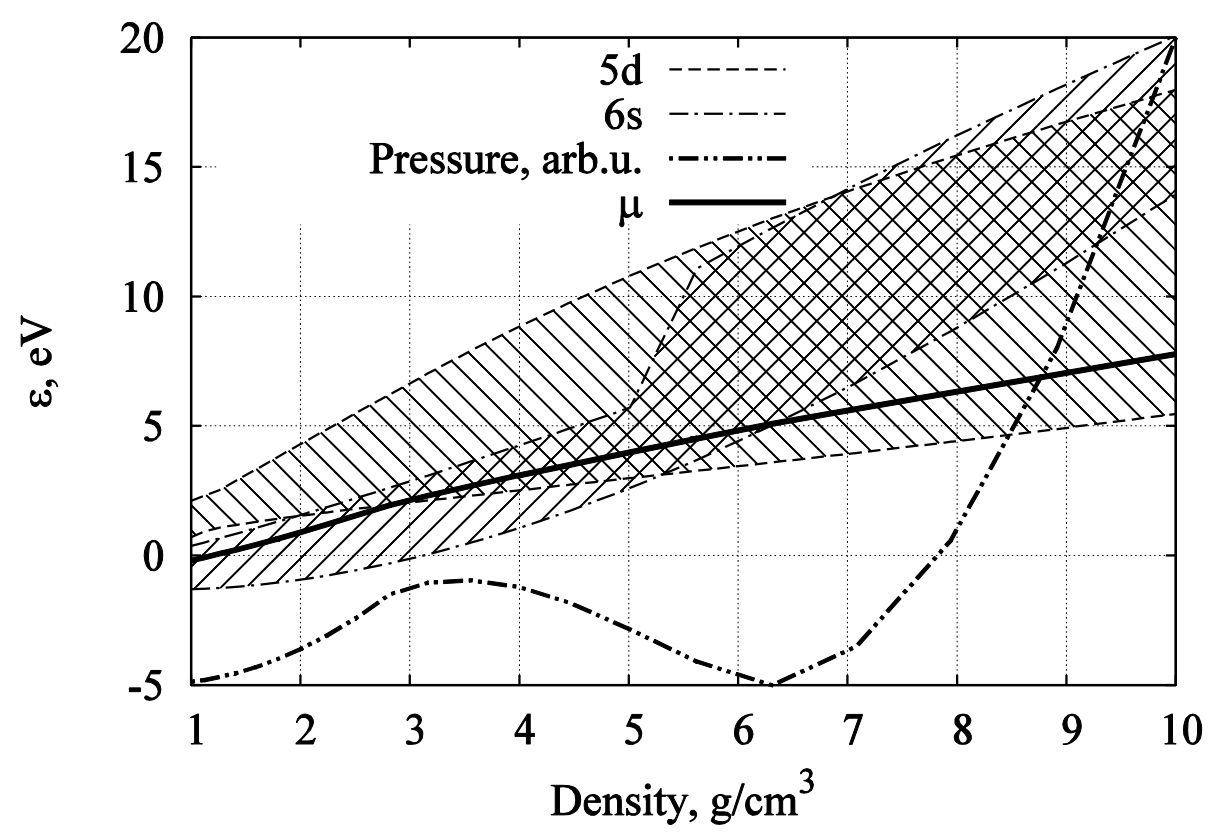

Fig. 11. Bands structure of $6 \mathrm{~s}$ and $5 \mathrm{~d}$ levels of cesium atom in dependence on the density. The solid curve is the Fermi level $(\mu)$, dashed curves are the edges of $5 \mathrm{~d}$ zone, dash-dotted curves are the edges of 6 s zone.

Dash-double-dot curve is the cold pressure in arbitrary units.

The figure 11 shows the two zones arising from discrete levels $6 \mathrm{~s}$ and $5 \mathrm{~d}$, the edges of which are indicated by dash-dotted lines and dashed lines, respectively. It is seen that at densities below $\approx 6 \mathrm{~g} / \mathrm{cm}^{3}$ electrons occupy the bottom of zone $6 \mathrm{~s}$ (corresponding energies lie below the Fermi level $\mu$ (solid line)). However, at densities greater than $6 \mathrm{~g} / \mathrm{cm}^{3}$, the lower part of the $5 \mathrm{~d}$ band becomes filled. The density at which this transition occurs corresponds to the minimum pressure on the curve of the cold pressure, which graph in arbitrary units is shown in Fig. 11 (dashdouble-dotted line). So the reason for the second minimum is connected with s-d transition of valence electron. At density $\rho \approx 2.9 \mathrm{~g} / \mathrm{cm}^{3}$ the $5 \mathrm{~d}$-band-edge sinks underneath the Fermi level and at this point the second derivative of pressure $d^{2} P / d \rho^{2}$ changes the sign. The next change of the $d^{2} P / d \rho^{2}$ sign occurs at density $\rho \approx 5.3 \mathrm{~g} / \mathrm{cm}^{3}$, where $5 \mathrm{~d}$-band-edge becomes below than $6 \mathrm{~s}$-band-edge. Further, at the density $6.2 \mathrm{~g} / \mathrm{cm}^{3}$ the 6s-band-edge becomes above the Fermi level and at this point the first derivative of the pressure $d P / d \rho$ changes the sign (see Fig. 11). There is a correlation with the findings about the features of the melting curve, discussed in Ref. [18].

\section{Conclusion}

This work underlines that previously detected anomaly in the melting curve of cesium associated with the transition of valence electron from $\mathrm{s}$ into $\mathrm{d}$ state, leads to the appearance of the first kind of phase transition in the region where cesium is in 
the ionized state. Ionization of the atoms is due to the overlap of electron energy bands formed blurry energy levels (pressure ionization). The accuracy of the results is confirmed by the fact that AA model provides acceptable accuracy in describing the conventional liquid-vapor transition in cesium. Critical parameters of the transition, calculated by this method are suitable in accordance with the measured values.

\section{References}

1. Fortov V.E. Extreme states of matter: on earth and in the cosmos : The frontiers collection. Extreme states of matter / V.E. Fortov OCLC: 845976746. Berlin: Springer, 2011. - 332 p.

2. Zubarev V.N. Impact compressibility of liquid nitrogen and solid carbon dioxide / V.N. Zubarev, G.S. Telegin // Doklady Akademii Nauk SSSR. - 1962. T. 142. - № 2. - C. 309.

3. Equation-of-state, shock-temperature, and electrical-conductivity data of dense fluid nitrogen in the region of the dissociative phase transition / W.J. Nellis [et al.] // The Journal of Chemical Physics. - 1991. - Vol. 94. - № 3. - P. 2244.

4. Ross M. Polymerization, shock cooling, and the high-pressure phase diagram of nitrogen / M. Ross, F. Rogers // Physical Review B. - 2006. - Vol. 74. - № 2.

5. Shock compression of liquid nitrogen at a pressure of $320 \mathrm{GPa} /$ R.F. Trunin [et al.] // JETP Letters. - 2008. - Vol. 88. - № 3. - P. 189-191.

6. Measurement of density, temperature, and electrical conductivity of a shockcompressed nonideal nitrogen plasma in the megabar pressure range / M.A. Mochalov [et al.] // Journal of Experimental and Theoretical Physics. - 2010. Vol. 110. - № 1. - P. 67-80.

7. Farjam M. Density-functional calculations of the electronic properties of metals by the spherical cellular method / M. Farjam, H.B. Shore // Physical Review B. - 1988. - Vol. 37. - № 3. - P. 1059-1066.

8. Nikiforov A.F. Quantum-statistical models of hot dense matter. Methods for computation opacity and equation of state. / A.F. Nikiforov, V.G. Novikov, V.B. Uvarov. - Switzerland: Birkhäuser, 2005. - 428 p.

9. Vorob'ev V.S. Communication: Shock adiabat of atomic nitrogen at megabar pressures / V.S. Vorob'ev, A.S. Grushin, V.G. Novikov // The Journal of Chemical Physics. - 2012. - Vol. 137. - № 3. - P. 31102.

10. Voropinov A.I. Electronic energy spectra and the equation of state of solids at high pressures and temperatures / A.I. Voropinov, G.M. Gandel'man, V.G. Podval'nyi // Soviet Physics Uspekhi. - 1970. - Vol. 13. - № 1. - P. 56-72. 
11. Götzlaff W. Thermopower- and Conductivity Behaviour Near the Gas-Liquid Critical Point of Mercury* / W. Götzlaff, G. Schönherr, F. Hensel // Zeitschrift für Physikalische Chemie. - 1988. - Vol. 156. - № Part_1. - P. 219-224.

12. Kozhevnikov V.F. Equation of state and sound speed of cesium at temperatures up to $2200 \mathrm{~K}$ and pressures up to $60 \mathrm{MPa} / \mathrm{V} . \mathrm{F}$. Kozhevnikov // Journal of Experimental and Theoretical Physics. - 1990. - Vol. 70. - № 2. - P. 298-310.

13. Bobrov V.B. The Kramers-Kronig relations for permittivity, "True" screening radius, and critical point of a coulomb system / V.B. Bobrov, S.A. Trigger // High Temperature. - 2011. - Vol. 49. - № 4. - P. 495-505.

14. Khomkin A.L. Phase transition into the metallic state in hypothetical (without molecules) dense atomic hydrogen / A.L. Khomkin, A.S. Shumikhin // Plasma Physics Reports. - 2013. - Vol. 39. - № 10. - P. 857-862.

15. Kennedy G.C. Fusion Curve and Polymorphic Transitions of Cesium at High Pressures / G.C. Kennedy, A. Jayaraman, R.C. Newton // Physical Review. - 1962. Vol. 126. - № 4. - P. 1363-1366.

16. Stishov S.M. Electrical Resistivity of Liquid Cesium at High Pressures / S.M. Stishov, I.N. Makarenko // Journal of Experimental and Theoretical Physics. 1968. - Vol. 27. - № 3. - P. 378-380.

17. Simozar S. Theoretical melting curve of caesium / S. Simozar, L.A. Girifalco // Journal of Physics F: Metal Physics. - 1983. - T. 13. - № 6. - C. 1145-1155.

18. Stishov S.M. The thermodynamics of melting of simple substances / S.M. Stishov // Soviet Physics Uspekhi. - 1975. - T. 17. - № 5. - C. 625-643.

19. Cannon J.F. Behavior of the Elements at High Pressures / J.F. Cannon // Journal of Physical and Chemical Reference Data. - 1974. - Vol. 3. - № 3. - P. 781.

20. Averill F.W. Calculation of the Cohesive Energies and Bulk Properties of the Alkali Metals / F.W. Averill // Physical Review B. - 1972. - Vol. 6. - № 10. P. 3637-3642.

21. Stishov S.M. Critical phenomena in metallic one-component liquids / S.M. Stishov // Physical Review B. - 1993. - Vol. 47. - № 18. - P. 12260-12262.

22. Khomkin A.L. Vapor-liquid (insulator-metal) phase transition in alkali metal vapors / A.L. Khomkin, A.S. Shumikhin // Journal of Experimental and Theoretical Physics. - 2014. - Vol. 118. - № 1. - P. 72-79. 


\section{Contents}

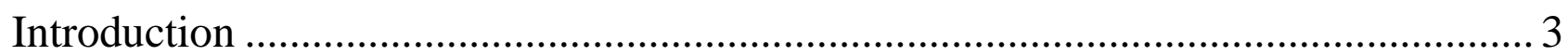

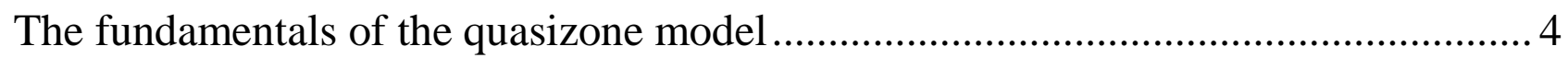

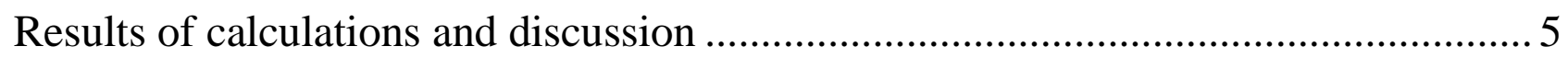

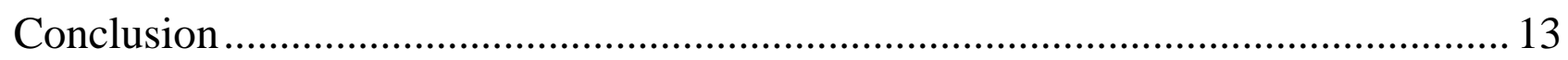

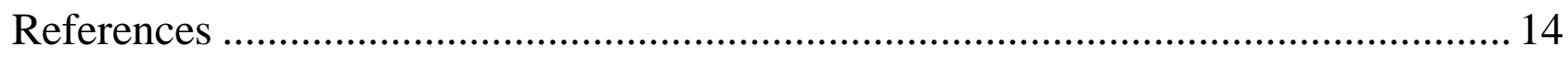

